\title{
Evaluación físico química de fruta de pitahaya (Selenicereus megalanthus) en diferentes estados de desarrollo
}

\author{
(Physical chemical evaluation of pitahaya fruit \\ (Selenicereus megalanthus) in different development stages)
}

\author{
Andrea Sotomayor, ${ }^{1}$ Soledad Pitizaca, ${ }^{1,2}$ Maritza Sánchez, ${ }^{1,3}$ Armando Burbano, ${ }^{1}$ Alejandra Díaz, ${ }^{1}$ \\ José Nicolalde, ${ }^{1}$ William Viera ${ }^{1}$, Carlos Caicedo ${ }^{1}$, Yadira Vargas ${ }^{1}$
}

\begin{abstract}
Resumen
En esta investigación se evaluaron los cambios físicos y químicos que ocurren durante el crecimiento y maduración de la fruta llamada pitahaya. Utilizando la tabla de color de pitahaya amarilla (NTC-3554), se colectaron frutos en los seis estados de madurez (0 verde oscuro a 6 completamente amarillo) para evaluar el peso del fruto; mientras que para las variables porcentaje de pulpa y cáscara, firmeza de la pulpa (N), sólidos solubles ( $\left.{ }^{\circ} \mathrm{Brix}\right)$, pH, acidez (\%) y la evaluación sensorial de sabor y apariencia, se tomaron frutos solamente en dos estados ( 0 y 6$)$. El porcentaje de cáscara disminuyó de 55.93 a 33.40 \%; mientras que el de pulpa aumentó de 44.07 a 66.60 \% entre el estado 0 y 6 , respectivamente. El fruto al llegar al estado 6 presentó menor firmeza de pulpa (6.20 N), acidez titulable (0.14 \%), y mayor contenido de sólidos solubles (20.74 ${ }^{\circ} \mathrm{Brix}$ ) y pH (4.86). Por otro lado, la prueba sensorial en los estados 0 y 6 fueron aceptados por el consumidor debido al alto contenido de grados Brix presentados en ambos estados.
\end{abstract}

\section{Palabras clave}

Calidad de fruta; madurez; peso fresco; grados Brix; acidez.

\begin{abstract}
In this research, physical and chemical changes that occur during growth and ripening of pitahaya fruit were assessed. Using the color chart of yellow pitahaya (NTC-3554), fruits were collected in the six stages of ripening (0 dark green to 6 completely yellow) to evaluate the weight of the fruit; while for the variables pulp and skin percentage, pulp firmness $(\mathrm{N})$, soluble solids $\left({ }^{\circ} \mathrm{Brix}\right), \mathrm{pH}$, acidity (\%) and sensory evaluation of flavor and appearance, fruits were only taken in two states (0 and 6). Percentage of skin decreased from 55.93 to $33.40 \%$, whereas that pulp increased from 44.07 to $66.60 \%$ among state 0 and 6, respectively. Fruit in the state 6 showed lower pulp firmness (6.20 N) and titulable acidity (0.14\%), and higher soluble solids content (20.74\%) and pH (4.86). On the other hand, in terms of sensory test, both states ( 0 and 6 ) were accepted by the consumer due to the high percentage of soluble solids.
\end{abstract}

\section{Keywords}

Fruit quality; ripening; fresh weight; Brix degrees; acidity.

\section{Introducción}

La pitahaya amarilla (Selenicereus megalanthus Haw.) es una cactácea silvestre, originaria de América Central y parte de Sudamérica, encontrándose distribuida en Bolivia, Perú, Colombia, Venezuela y Ecuador (Morillo et al., 2017). Se cultiva entre 500 y 1900 msnm, a una temperatura entre 18 y $25^{\circ} \mathrm{C}$, pluviosidad que fluctúa entre 1200 y $2500 \mathrm{~mm}$ año-1 y humedad relativa entre 70 y 80 \% (Vásquez-Castillo et al., 2016).

En Ecuador, se cultiva la pitahaya roja (Hylocereus undatus Britt et Rose) y la pitahaya amarilla, esta última también es cultivada en Colombia e Israel, siendo atractiva por su aparien-

\footnotetext{
1 Instituto Nacional de Investigaciones Agropecuarias (INIAP) \{andrea.sotomayor, gabriela.pitizaca, martiza.sanchez, armando.burbano, alejandra.diaz, jose.nicolalde, william.viera, carlos.caicedo, yadira.vargas\} @iniap.gob.ec

2 Instituto Superior Tecnológico Crecermas

3 Universidad Politécnica de Chimborazo
} 
cia externa, y por ser considerada como una de las frutas más exóticas del mundo. La fruta se caracteriza por tener una corteza de color amarillo con espinas y una pulpa blanca aromática con pequeñas semillas negras. Por otro lado, la pitahaya roja cultivada, principalmente en México, Nicaragua y Vietnam, se diferencia por contar con la presencia de brácteas en lugar de espinas; su pulpa puede ser blanca o roja clara (dependiendo de la variedad), con pequeñas semillas negras (Rodríguez et al., 2005).

En Ecuador, existen dos ecotipos de la pitahaya amarilla: la primera denominada "Pichincha", también conocida como "Nacional" (sus frutos alcanzan hasta los $150 \mathrm{~g}$ de peso), se cultiva en el noroccidente de Pichincha; y la segunda conocida como el ecotipo "Palora" (sus frutos alcanzan hasta los $350 \mathrm{~g}$ de peso), que se cultiva en grandes extensiones en el cantón Palora (Morona Santiago) y, en menor superficie, en Pichincha (Trujillo, 2014).

Esta fruta es considerada como exótica por sus características, como sabor, apariencia y calidad; posee vitamina C, fibra, carbohidratos y agua en un 80 \%. Puede ser consumida en fresco o en jugos, cocteles, helados, yogurt y mermeladas; además, el aceite de sus semillas tienen efecto laxante y ayuda a disminuir el colesterol en la sangre (Guzmán et al., 2012 y Trujillo, 2014). Es conocida por aliviar los problemas estomacales, endocrinógenos y también mejora el funcionamiento del tracto digestivo (Huachi et al., 2015); el beneficio más conocido es su capacidad antioxidante que se le atribuye porque sus semillas tienen un alto contenido de ácidos grasos naturales, en especial el ácido linoléico (Chemah et al., 2010), debido a que este funciona en el organismo como buffer capturando el colesterol, generando un efecto cardiotónico (Omidizadeh et al., 2011).

En Ecuador, la información sobre la calidad del fruto de pitahaya es escasa, sin embargo, se ha determinado que los frutos de pitahaya ecotipo Palora maduros presentan valores altos en firmeza, acidez titulable, sólidos solubles, pH, vitamina C, capacidad antioxidante y polifenoles totales; siendo la mayor cantidad de fruta exportable con mínimas pérdidas poscosecha (1.5 \%) (Bolaños y Calero, 2015, Vásquez-Castillo et al., 2016).

El objetivo de esta investigación fue realizar la evaluación de los cambios físico-químicos en los estados de desarrollo de la fruta de pitahaya ecotipo Palora con la finalidad de generar información sobre este frutal de exportación.

\section{Metodología}

Los frutos evaluados se tomaron de un huerto comercial ubicado en el cantón Palora, provincia de Morona Santiago, Ecuador. Las plantas de pitahaya tenían 16 años de edad y estuvieron establecidas en un sistema de tutores inertes. La recolección de los frutos fue en el periodo de producción entre diciembre de 2017 y enero del 2018. Durante el período de evaluación, se registró una temperatura de 20 a $24^{\circ} \mathrm{C}$, con un promedio de $22.5^{\circ} \mathrm{C}$; mientras que la precipitación fue de $296 \mathrm{~mm}$ en diciembre y $309 \mathrm{~mm}$ en enero.

Para llevar a cabo está investigación, se seleccionaron frutos, según la tabla de color de la Norma Técnica Colombiana (ICONTEC, 1996), con escalas de madurez de 0 al 6 (Figura 1), siendo 0 verde-oscuro y 6 totalmente amarillo. 
Figura 1. Tabla de color de pitahaya amarilla, según la Norma Técnica Colombiana NTC- 3554,1996

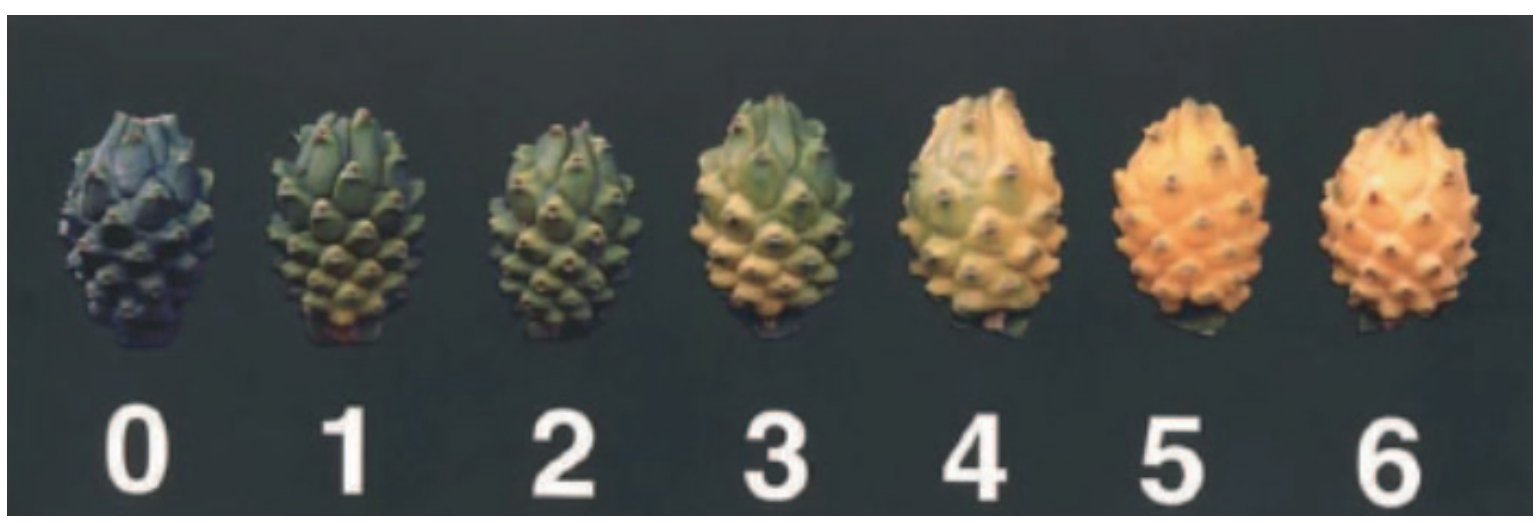

Se colectaron 15 frutos en cada estado para registrar las variables peso fresco (g), mientras que para las variables de porcentaje de pulpa y cáscara, firmeza de la pulpa (N), sólidos solubles ( $\left.{ }^{\circ} \mathrm{Brix}\right), \mathrm{pH}$, acidez (\%) y evaluación sensorial se tomaron 15 frutos del estado 0 y 6 , respectivamente. El peso fresco se registró utilizando una balanza digital (Citizen Scale, modelo CG 4102C; precisión de $0.01 \mathrm{~g}$ ). La firmeza se determinó con un penetrómetro manual (Force Gauge, modelo GY-4) provisto con un puntal de $3.50 \mathrm{~mm}$ de diámetro, realizando dos lecturas en la parte ecuatorial del fruto, y los resultados se expresaron en Newtons (N). El contenido de sólidos solubles totales ( ${ }^{\circ} \mathrm{Brix}$ ) se midió utilizando un refractómetro digital (Hanna instruments, modelo Hi 96801, USA), el pH con un potenciómetro (BOECO, modelo PT-380) y la acidez titulable (expresada como \% ácido cítrico); todas las variables se midieron con la metodología descrita por la AOAC (2012).

Se utilizó un diseño completo al azar (DCA), con tres repeticiones. Los análisis se realizaron mediante el paquete estadístico Infostat versión 2015 (Di Renzo et al., 2015). Se recurrió a la prueba de Tukey con nivel de significancia al $5 \%$ para determinar las diferencias estadísticas entre las medias de los tratamientos.

Adicionalmente, se realizó un análisis sensorial con frutos en estado de madurez de 0 y 6. Se contó con un panel no entrenado de 12 personas, donde cada uno degustó una porción de pulpa para valorar el sabor y la aceptación general del fruto (color externo y apariencia de las brácteas) mediante escalas hedónicas de intervalos (Centurión et al., 2008). La escala utilizada correspondió a: 1 = me desagrada mucho, 2 = me desagrada ligeramente, 3 = ni me gusta ni me disgusta, 4 = me gusta ligeramente, 5 = me gusta mucho .

\section{Resultados}

Se pudo observar diferencias estadísticas $(p<0.01)$ en todas las variables evaluadas. El peso fresco de la fruta aumentó conforme avanzaron los distintos estados fenológicos, iniciándose con un peso de $262.11 \mathrm{~g}$ (estado de madurez 0) e incrementándose a $394.66 \mathrm{~g}$ (estado de madurez 6) como se puede ver en la Figura 2. 
Figura 2. Cambios en peso fresco en los 6 estados de maduración

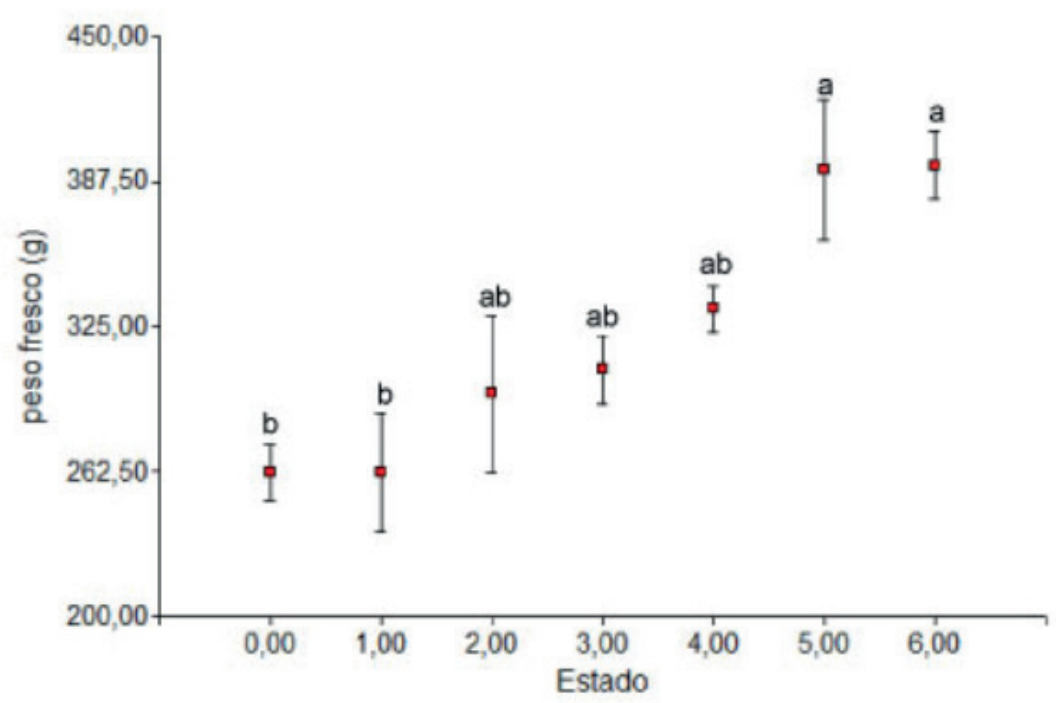

El porcentaje de cáscara, disminuyó conforme avanzó el estado de madurez, en el estado 0 se obtuvo un valor de $55.93 \%$ en comparación al estado 6 con $33.40 \%$, mientras que el porcentaje de pulpa aumentó desde un 44.07 \% en el estado 0 hasta llegar al $66.60 \%$ en el estado 6 (Tabla 1). En firmeza de pulpa se observó que existe diferencia significativa entre los dos estados de maduración de los frutos, donde el estado 0 presentó 17.77 N y el estado 66.20 N. En el contenido de sólidos solubles ( ${ }^{\circ}$ Brix), se encontró que esta variable aumentó de 18.34 a 20.74 ( ${ }^{\circ} \mathrm{Brix}$ ) del estado 0 al 6, respectivamente. Respecto a la acidez titulable, expresada como contenido de ácido cítrico, disminuyó significativamente desde 0.26 \% en estado 0 a $0.14 \%$ en estado 6 . Se encontró diferencia estadística para $\mathrm{pH}$, donde el estado 0 presentó un valor de 4.56 y el estado 6 en valor de 4.86 (Tabla 1).

Tabla 1. Medias de las variables registradas de frutos cultivar Palora en los estado 0 y 6

\begin{tabular}{|l|l|l|}
\hline \multicolumn{1}{|c|}{ Variable } & \multicolumn{1}{|c|}{ Estado } & \multicolumn{1}{c|}{ Medias } \\
\hline \multirow{2}{*}{ Pulpa (\%) } & 0 & $44.07 \pm 7.38 \mathrm{~b}$ \\
\cline { 2 - 3 } & 6 & $66.60 \pm 1.72^{\mathrm{a}}$ \\
\hline \multirow{2}{*}{ Cáscara (\%) } & 0 & $55.93 \pm 7.38 \mathrm{a}$ \\
\cline { 2 - 3 } & 6 & $33.40 \pm 1.72 \mathrm{~b}$ \\
\hline \multirow{2}{*}{ Firmeza de pulpa (N) } & 0 & $17.77 \pm 2.04 \mathrm{a}$ \\
\hline \multirow{2}{*}{ Sólidos solubles (\%) } & 6 & $6.20 \pm 1.52 \mathrm{~b}$ \\
\hline \multirow{2}{*}{ Acidez titulable (\%) } & 0 & $18.34 \pm 3.54 \mathrm{~b}$ \\
\cline { 2 - 3 } & 6 & $20.74 \pm 0.73 \mathrm{a}$ \\
\hline \multirow{2}{*}{ pH } & 0 & $0.26 \pm 0.07 \mathrm{a}$ \\
\cline { 2 - 3 } & 6 & $0.14 \pm 0.01 \mathrm{~b}$ \\
\hline
\end{tabular}

En relación al sabor, los datos del análisis sensorial mostraron que el estado 0 obtuvo un valor promedio de 3.82 (me gusta ligeramente) en la escala de evaluación; mientras que el es- 
tado 6 con 4.67 me gusta mucho, lo cual está relacionado al alto contenido de sólidos solubles encontrado en ambos estados. En el caso de la aceptación general (color externo y apariencia de las brácteas), el estado 0 obtuvo un valor promedio de 3.43 (ni me gusta ni me disgusta); mientras que en el estado 6 alcanzó un valor mejor de aceptación de 4.50 (me gusta mucho), lo cual estaría relacionado a la coloración amarilla del fruto, que constituye un atractivo visual para el consumidor.

\section{Discusión}

En la variable peso de fruto existió un incremento conforme avanza el estado de maduración. En estudios realizados con el mismo ecotipo se ha reportado un peso de $331.60 \mathrm{~g}$ (Vásquez-Castillo et al., 2016) en una fruta cosechada en estado 5; este valor es menor a lo reportado en este estudio en el mismo estado (392,93 g). Campos-Rojas et al. (2011) mencionan que las pitahayas amarillas presentan un tamaño intermedio en comparación a las rojas, que va entre 350 y 469 g en México (Centurión et al., 2008), de 415 a 534 g en Brasil (Brunini y Cardoso, 2011), de 425 a $550 \mathrm{~g}$ en Israel (Nerd et al., 1999), y de 141 a $397 \mathrm{~g}$ en Florida EE. UU. (Crane y Balerdi, 2005).

El porcentaje de pulpa aumentó de manera acelerada desde el estado 0 al 6 llegando a $66.60 \%$, resultado que supera al reportado por Cañar et al. (2014) con un valor de 62.64 \% para pitahayas amarillas con madurez comercial. Esta tendencia también se observa en estudios realizados por Centurión et al., (2008), donde mencionan que durante el cambio en color ocurre una importante acumulación de la porción comestible (pulpa) del fruto, y una disminución de la proporción cáscara cuyo mecanismo metabólico aún no se ha determinado.

Por otro lado, el porcentaje de cáscara fue menor en el estado 6 comparado con el estado 1, resultados con valores cercanos a los reportados por Guerrero (2014) quien reportó que la fruta en estado 0 obtuvo 54.60 \%; mientras que en estado 6 obtuvo $41.20 \%$. En pitahaya roja, Nerd et al. (1999) mencionan que este porcentaje disminuye de 57.50 a $32.50 \%$ de fruta inmadura a madura.

La firmeza de la pulpa disminuye mientras avanza la madurez del fruto, la firmeza de los frutos representa el 79 \% de la consistencia inicial, valor superior a los reportados por Guerreo (2014) y Centurión et al. (2008), quienes manifiestan que la firmeza de los frutos al final de la evaluación representó el 35.20 y 67 \% de la consistencia inicial, respectivamente. La reducción de la firmeza en los frutos durante la maduración posiblemente se debe a la degradación de las paredes celulares, que contienen sustancias pépticas, provocando disminución de la rigidez y debilitamiento de la adhesión intercelular (Brummell, 2006).

El contenido de sólidos solubles es una característica muy apreciada en los frutos de pitahaya, debido a que este parámetro determina el dulzor de la fruta (Vásquez-Castillo et al., 2016). En este estudio se encontraron valores altos de sólidos solubles en ambos estados (0 y 6), resultado similar al reportado por Vásquez-Castillo et al. (2016) quienes mencionan un valor de $20.10{ }^{\circ}$ Brix en la fruta madura para el ecotipo Palora y $17.9^{\circ} \mathrm{Brix}$ para el ecotipo Nacional. Las pitahayas amarillas poseen un alto contenido de azúcares en comparación con las pitahayas rojas, estas últimas presentan valores entre 12 y 13 'Brix (Merten (2003) y Centurión et al., 2008) y entre 16 y 17 \% (Nerd et al., 1999). La acumulación de azúcares durante la maduración de los frutos de pitahaya se relaciona con una disminución en el contenido de almidón y mucílagos de la pulpa (Barrera y Nobel, 2004) y las condiciones climáticas y agronómicas (Guerrero, 2014). Yi-Lu et al., (2011) mencionan que la pitahaya en verano alcanza un valor de 19.6 ${ }^{\circ} \mathrm{Brix}$, mientras que en invierno el valor llega a $20.8^{\circ} \mathrm{Brix}$. 
La acidez titulable disminuyó significativamente desde el estado 0 al estado 6. VásquezCastillo et al. (2016) obtuvieron una acidez titulable de $0.12 \%$ en frutos maduros, resultados similares al de este estudio. Centurión et al. (2008) señalaron que la acidez titulable disminuye en los últimos 5 días de maduración de los frutos; por lo tanto, la diferencia de solo un día entre cosechas puede generar diferencias de acidez.

El pH aumentó desde el estado 0 al 6; resultado similar al reportado por García y Robayo (2008) donde fruta inmadura (estado 0) obtuvo un pH de 4.05, mientras que fruta madura presenta un valor de 4.7 (Vásquez-Castillo et al., 2016).

El análisis sensorial mostró una aceptación de esta fruta por parte de los panelistas en ambos estados de evaluación, resultado que se atribuye a que la pitahaya Palora presentó un alto contenido de sólidos solubles desde el estado 0, incrementándose el contenido de azúcares con la maduración, lo que hace que la fruta sea aceptable para el consumidor. En el estudio realizado por Centurión et al. (2008), el sabor de los frutos alcanzó una calificación superior considerada dulce con fruta madura.

La aceptación por color externo y apariencia del fruto en los estados 0 y 1 fueron los menores ya que 'ni me gusta ni me disgusta', mientras que en el estado 6 la tendencia fue 'me gusta mucho'. Valores similares fueron reportados por Centurión et al. (2008) donde los fruto de escalas inferiores tuvieron menor aceptación que los de escalas superiores que alcanzaron mayor nivel de preferencia. Además, con estos resultados, se infiere que la coloración amarilla del fruto en el último estado de maduración incide en la aceptación del consumidor.

\section{Conclusiones}

Los resultados de este estudio muestran que el peso fresco de la fruta de pitahaya aumenta conforme avanza el desarrollo (maduración) del fruto, llegando a obtener un peso aceptable para el mercado de exportación. El porcentaje de cáscara y pulpa son inversamente proporcionales en el desarrollo del fruto, es decir, el fruto inmaduro tiene un porcentaje de cáscara mayor y de pulpa menor, pero cuando la fruta llega a la madurez, el porcentaje de cáscara disminuye y el porcentaje de pulpa aumenta. El fruto al llegar a madurez presenta menor firmeza de pulpa y acidez titulable, mayor contenido de sólidos solubles y pH. Finalmente, los frutos en estado 6 de madurez tuvieron la mayor aceptación tanto para color externo como para la apariencia de brácteas; sin embargo en relación al sabor (balance dulzor y acidez), ambos estados ( 0 y 6 ) fueron aceptados por el alto contenido de sólidos solubles que presentaron.

\section{Agradecimientos}

Se agradece a la Sra. Noemi Ortiz, dueña de la Finca el Progreso por provisionar fruta para la realización de este estudio, como también al INIFAP por la capacitación prestada a los técnicos en frutales no tradicionales como pitahaya, y a INIAP por el financiamiento de esta investigación.

\section{Referencias}

Association of Official Analytical Chemists (AOAC). (2012). Official Methods of Analysis. AOAC International, Washington.

Barrera, D., Nobel, S. (2004) Carbon and water relation for development fruits of Opuntia ficus-indica (L.) Miller, including effects of drought and gibberellic acid. J. Exp. Bot., 55,719-729. 
Bolaños, G., Calero, C. (2015). Calidad y componentes bioactivos de pitahaya (Hylocereus triangularis) y guayaba (Psidium guajava) debido a índices de madurez y temperatura de conservación. Tesis de pregrado. Universidad de las Fuerzas Armadas.

Brummell, D. (2006). Cell wall disassembly in ripening fruit. Functional Plant Biology, 33, 103-119.

Brunini, M., Cardoso, S. (2011). Qualidade de pitaias de polpa branca armazenadas em diferentes temperaturas. Revista Caatinga, Mossoró, 24, 78-84.

Campos-Rojas R., Pinedo-Espinoza, J., Campos-Montiel, R., y Hernández-Fuentes, A. (2011). Evaluación de plantas de pitaya (Stenocereus spp.) de poblaciones naturales de Monte Escobedo, Zacatecas. Rev. Chapingo Ser. Hortic., 17 (3), 173-182.

Cañar, D., Caetano, C., y Bonilla, M. (2014). Caracterización fisicoquímica y proximal del fruto de pitahaya amarilla [Selenicereus megalanthus (k. schum. ex vaupel) moran] cultivada en Colombia. Revista Agronomía, 22 (1), 77-87.

Centurión, A., Pérez, S., Solís, S. Báez, R., Mercado S. E., Saucedo, V., y Sauri D. (2000) Crecimiento, desarrollo y comercialización de la pitahaya (Hylocereus undatus) durante la postcosecha. Rev. Iberoam. Tecnol. Postcos, 2, 161-168.

Centurión, A., Solís, S., Saucedo, C., Báez, R., y Sauri, D. (2008). Cambios físicos, químicos y sensoriales en frutos de pitahaya (Hylocereus undatus) durante su desarrollo. Rev. Fitotec. Mex., 31 (1), 1-5.

Chemah, T., Aminah, A., Noriham, A.y Aída, W. (2010). Determination of pitaya seeds as a natural antioxidant and source of essential fatty acids. International Food Research Journal, 17, 1003-1010.

Crane, J., Balerdi, C. (2005). The Pitaya (Hylocereus undatus and other spp.) in Florida. Trop. Res. Educ. Ctr., Univ. of Florida, Homestead.

Di Rienzo, J., Macchiavelli, R., Casanoves, F. (2011). Modelos lineales mixtos: aplicaciones en InfoStat (1a. ed). Córdoba, Argentina: Editorial Brujas.

García, M., Robayo, P. (2008). Evaluación del uso de atmósferas modificadas pasivas y temperaturas bajas en la conservación de pitaya amarilla (Selinicereus megalanthus Shuman). Revista Corpoica- Ciencia y Tecnología Agropecuaria, 9 (1), 30-39.

Guzmán, O., Pérez, L. y Patiño, A. (2012). Reconocimiento de nemátodos fitoparásitos en pitahaya amarilla (Selenicereus megalanthus HAW.). Boletín Cientifico Centro de Museos, 16(2), 149-161.

Guerrero, M. (2014). Estudio del manejo poscosecha de pitahaya amarilla (Selenicereus megalanthus) procedente del cantón Pedro Vicente Maldonado de la provincia de Pichincha. Tesis de pregrado. Escuela Politécnica Nacional.

Huachi, L., Yugsi, E., Paredes, M., Coronel, D., Verdugo, K., y Coba Santamaría, P. (2015). Desarrollo de la pitahaya (Cereus sp.) en Ecuador. La Granja: Revista de Ciencias de la Vida. 22(2), 50-58.

Instituto Colombiano de Normas Técnicas y Certificación (ICONTEC). (1996). Norma Técnica Colombiana. NTC 3554. Frutas frescas. Pitahaya amarilla. Bogotá: ICONTEC: 1-14

Jiménez, L., González, M., Cruz, S., Santana R. y Villacís, L. (2017). Análisis poscosecha de frutos de pitahaya amarilla (Cereus triangularis Haw.), a distintos niveles de madurez y temperatura. J. Selva Andina Biosph. 2(5), 107-115.

Merten, S. (2003). A Review of Hylocereus production in the United States. J. Prof. Assoc. Cactus Develop, 1, 98-105.

Morillo, A., Tovar, Y., y Morillo, Y. (2017). Caracterización molecular de la pitahaya amarilla (Selenicereus megalanthus Haw.) en la provincia de Lengupá, Boyaca-Colombia. Biotecnología en el Sector Agropecuario y Agroindustrial, 15 (1),11-18.

Nerd, A., Gutman, F., y Mizrahi, Y. (1999). Ripening and postharvest behaviour of fruits of two Hylocereus undatus species (Cactaceae). Postharv. Biol. Technol., 17, 39-45.

Omidizadeh, A., Mohd, R., Ismael, A., Roohinejad, S., Nategui, L., Abu, M. (2011). Cardioprotective compounds of red pitaya (Hylocereus polyrhizus) fruit. Journal of Food, Agriculture \& Environment, 9 (3-4), 152-156. 
Osuna Enciso, T., Ibarra Zazueta, M., Muy Rangel, M., Valdez Torres, J., Villarreal Romero, M., Hernández Verdugo, S. (2011). Calidad postcosecha de frutos de pitahaya (Hylocereus undatus Haw.) cosechados en tres estados de madurez. Revista fitotecnia mexicana, 34 (1), 63-72.

Rodríguez, D., Patiño, M., Miranda, D., Fischer, G., y Galvis, J. (2005). Efecto de dos índices de madurez y dos temperaturas de almacenamiento sobre el comportamiento en almacenamiento sobre el comportamiento en poscosecha de la pitahaya amarilla (Selenicereus megalanthus Haw.). Rev. Fac.Nal.Agr. Medellín, 58 (2), 2827-2857.

Vásquez-Castillo, W., Aguilar, K., Vilaplana, R., Viteri, P., Viera, W., y Valencia-Chamorro, S. (2016). Calidad del fruto y pérdidas poscosecha de pitahaya amarilla (Selenicereus megalanthus Haw.) en Ecuador. Agronomía Colombiana, 34 (1Supl.), S1081-S1083.

To, L.; Ngu, N.; Duc, N.; Huong, H. (2002). Dragon fruit quality and storage life: Effect of harvest time, use of plant growth regulators and modified atmosphere packaging. Acta Hort., 575,611-621.

Trujillo, D. (2014). Micoorganismos asociados a la pudrición blanda del tallo y manchado del fruto en el cultivo de pitahaya amarilla (Tesis de pregrado). Universidad Central del Ecuador.

Yi-Lu, J., Tzong-Shyan, L., y Ching-Lung, L. (2011). Phenology, canopy composition, and fruit quality of yellow pitaya in tropical Taiwan. HortScience, 46 (11), 1497-1502. 